\title{
Perfil epidemiológico dos pacientes com fraturas torácicas e lombares tratadas cirurgicamente no Serviço de Neurocirurgia do Hospital de Base do Distrito Federal (Brasília-Brasil)
}

\author{
Cléciton Braga Tavares', Emerson Brandão Sousa', Igor Brenno Campbell Borges', \\ Amauri Araújo Godinho Júnior'2, Nelson Geraldo Freire Neto ${ }^{3}$
}

Unidade de Neurocirurgia do Hospital de Base do Distrito Federal, Brasília, DF, Brasil.

\section{RESUMO}

Objetivos: Apresentar o perfil epidemiológico e os fatores de risco para déficit neurológico de 52 pacientes com fratura traumática da coluna torácica e lombar tratados cirurgicamente no Serviço de Neurocirurgia do Hospital de Base do Distrito Federal. Métodos: Trata-se de trabalho retrospectivo de pacientes com fratura da coluna torácica e lombar tratados cirurgicamente no período de julho de 2007 a julho de 2012. Resultados: Predomínio do sexo masculino (78,8\%); faixa etária mais comum é $20-40$ anos (57,6\%); segmento fraturado: T1-T10 (19,2\%), T11-L2 (61,5\%), L3-L5 (19,3\%); 48\% dos indivíduos tinham déficit neurológico; fratura tipo A é mais comum (42,3\%); mecanismo do trauma: acidente automobilístico (23\%), queda de altura (42,3\%), motociclístico (26,9\%); predomínio da fratura tipo C no segmento torácico 45,4\% e tipo A no lombar $63,3 \%(p<0,01)$; déficit neurológico: torácico $81,8 \%$, lombar 23,3\% ( $p<0,05)$; déficit neurológico toracolombar: tipo $A 31,8 \%$, tipo $B 47,3 \%$, tipo $C$ $81,8 \%(p=0,02)$. Conclusão: A maioria dos pacientes era do sexo masculino e adulto jovem. Cerca de $48 \%$ apresentavam déficit à admissão hospitalar e tinham a junção T11-L2 como principal local de ocorrência. A fratura tipo A da AO foi mais encontrada entre as lesões lombares e a tipo $C$ entre as torácicas. O principal mecanismo do trauma foram os acidentes de trânsito. As fraturas torácicas e as lesões do tipo C são fatores de risco para lesão neurológica.

\section{PALAVRAS-CHAVE}

Coluna vertebral, fraturas da coluna vertebral/epidemiologia, vértebras torácicas/lesões, vértebras lombares/lesões, traumatismo da coluna vertebral/epidemiologia.

\begin{abstract}
Epidemiological profile of patients with thoracic and lumbar fractures surgically treated in Neurosurgery Service at Hospital de Base do Distrito Federal (Brasilia, Brazil)

Objective: We present the epidemiological and risk factors for neurological deficit of 52 patients with traumatic fracture of the thoracic and lumbar spine were surgically treated in the neurosurgery service at the Hospital de Base do Distrito Federal, Brasilia-Brazil. Methods: This was a retrospective study of patients with fractures of the thoracic and lumbar spine treated surgically in the period July 2007 to July 2012. Results: Predominantly male (78.8\%) is the most common age group 20-40 years (57.6\%); fractured segment: T1-T10 (19.2\%), T11-L2 (61.5\% ) L3-L5 (19.3\%), 48\% of subjects had neurologic deficit; fracture type $A$ is the most common (42.3\%), mechanism of injury: motor vehicle accidents (23\%), falls (42.3\%), motorcycle (26.9\%); predominance of type $C$ fractures in the thoracic segment $(45.4 \%)$ inin lumbar type $A$ (63.3\%) ( $p<0.01)$; neurological deficit: $81.8 \%$ thoracic, lumbar $23.3 \%(p$ $<0.05)$; neurologic deficit thoracolumbar: $31.8 \%$ type $A$, type $B 47.3 \%$ and $81.8 \%$ type $C(p=0.02)$. Conclusion: Most patients were male and young adult. About $48 \%$ had deficits on admission and had the junction T11-L2 as the main place of occurrence. The fracture of $A O$ type $A$ was more frequently found among back injury and type $C$ between chest. The main mechanism of injury were traffic accidents. Chest injuries and type $C$ fractures are risk factors for neurological injury.
\end{abstract}

1 Médico-residente de Neurocirurgia do Hospital de Base do Distrito Federal, Brasília, DF, Brasil.

2 Médico neurocirurgião e preceptor do Programa de Residência Médica em Neurocirurgia do Hospital de Base do Distrito Federal, Brasília, DF, Brasil.

3 Médico neurocirurgião, preceptor do Programa de Residência Médica e chefe da Unidade de Neurocirurgia do Hospital de Base do Distrito Federal, Brasília, DF, Brasil. 


\section{KEYWORDS}

Spine, spinal fractures/epidemiology, thoracic vertebrae/injuries, lumbar vertebrae/injuries, spinal injuries/epidemiology.

\section{Introdução}

As fraturas da coluna torácica e lombar são as mais frequentes do esqueleto axial e correspondem a cerca de $89 \%$ das fraturas da coluna vertebral, segundo alguns autores.

Dois terços dessas fraturas ocorrem na junção toracolombar, entre T11-L2 (50\% das fraturas da coluna torácica no nível T12 e 60\% das fraturas da coluna lombar no nível L1). A coluna torácica entre T1-T10 corresponde a um segmento relativamente mais rígido entre dois móveis que são a coluna cervical e lombossacra. ${ }^{1,2}$

A coluna torácica apresenta cifose no plano sagital e possui maior rigidez pela configuração de suas estruturas anatômicas e também pela estabilidade adicional proporcionada pela articulação com as costelas e o esterno, e isso quadriplica a sua resistência à compressão, aumenta sua resistência à extensão em 70\%, enquanto a resistência à flexão e a rotação são menos significativas.

Nos últimos anos vem se observando um aumento no número de pacientes vítimas de lesão na coluna torácica e lombar, sendo elevado o índice de morbidade e mortalidade. ${ }^{3}$

Estudos mostram que 40,1 habitantes dos Estados Unidos, em cada grupo de 1 milhão, sofrem trauma da coluna torácica ou lombar a cada ano. Cunha et al. ${ }^{4}$ apresentaram uma incidência de 11,8 lesões traumáticas nessa topografia por milhão de habitantes na região metropolitana de Belo Horizonte. Essas fraturas possuem grande impacto na vida do paciente e de suas famílias. Acarretam elevado custo ao sistema de saúde pública, incluindo internações, cirurgias e reabilitação, associadas à perda da produtividade do indivíduo, algumas vezes definitivamente. ${ }^{5}$

Apresentamos neste trabalho o perfil epidemiológico e os fatores de risco para déficit neurológico de 52 (cinquenta e dois) pacientes com fratura traumática da coluna torácica e lombar tratados cirurgicamente no Serviço de Neurocirurgia do Hospital de Base do Distrito Federal. É um serviço de referência, da capital federal e do centro-oeste brasileiro, no tratamento do traumatismo raquimedular, com realização média de 100 cirurgias/ano para correção de fraturas vertebrais. Os dados levantados são importantes do ponto de vista de saúde pública e gestão de recursos.

\section{Material e métodos}

Trata-se de um trabalho retrospectivo, baseado na revisão de prontuários médicos, de pacientes com fratura da coluna torácica e lombar tratados cirurgicamente no Serviço de Neurocirurgia do Hospital de Base do Distrito Federal, Brasília-Brasil, no período de julho de 2007 a julho de 2012 .

Nesse intervalo de tempo foram operados no hospital 515 pacientes com fratura vertebral, sendo 200 cervicais (31\%) e 315 torácicas e lombares (69\%).

Os dados levantados foram: faixa etária, sexo, mecanismo de trauma, vértebras fraturadas, tipo de fratura (baseado na classificação da Magerl/AO) e déficit neurológico (baseado na classificação ASIA/Frankel). Os prontuários que não apresentavam todos esses dados no relatório de alta hospitalar foram excluídos do trabalho; ao todo, 263 indivíduos.

Os pacientes foram agrupados quanto à idade em cinco faixas etárias: 0-10 anos, 10-20 anos, 20-40 anos, 40-60 anos e acima de 60 anos. Os mecanismos do trauma foram divididos em 10 grupos: acidentes automobilísticos, acidentes motociclísticos, queda de altura, queda da própria altura, lesão por arma de fogo, mergulho em águas rasas, acidentes ciclísticos, acidente com máquina agrícola, agressão física e queda de material pesado sobre o corpo.

A classificação das fraturas utilizada foi a de Magerl et al., ${ }^{6}$ adotada pelo grupo $\mathrm{AO}$, que agrupa as fraturas em três grandes tipos: A - lesões por compressão do corpo vertebral; B - lesões por distração dos elementos anterior e/ou posterior; e C - lesões tipo A ou B com rotação e luxações complexas. Cada tipo, por sua vez, é subdividido em três subtipos: 1, 2 e 3 . E cada subtipo é dividido em três subgrupos: 1,2 e 3 . No presente estudo, optou-se por restringir a classificação aos tipos.

A avaliação da deficiência neurológica foi baseada na escala de Frankel et al., ${ }^{7}$ que foi modificada pela ASIA (American Spine Injury Association) e consiste em cinco graus de incapacidade: (A) Lesão completa, não existe função motora ou sensitiva nos segmentos sacrais S4-S5; (B) Lesão incompleta, preservação da sensibilidade e perda total da força motora abaixo do nível neurológico, estendendo-se até os segmentos sacrais S4-S5; (C) Lesão incompleta, função motora é preservada abaixo do nível 
neurológico, e a maioria dos músculos-chave possuem força grau 1 ou 2; (D) Lesão incompleta, função motora é preservada abaixo do nível neurológico, e a maioria dos músculos-chave possuem força grau 3 ou 4; e (E) Normal, sensibilidade e motricidade normais, grau 5.

A gradação da força muscular foi realizada de acordo com o método do Medical Research Council. Grau 0: Paralisia total; Grau 1: Contração fracamente detectável; Grau 2: Força insuficiente para atuar contra gravidade; Grau 3: Força suficiente para atuar contra a gravidade; Grau 4: Força presente, porém ainda não é normal; Grau 5: Força normal. ${ }^{8}$

As informações foram compiladas em uma planilha do programa Excel-Windows, perfazendo um total de cinquenta e dois indivíduos $(\mathrm{n}=52)$. Todos os dados foram analisados pelo software Statistical Package for the Social Sciences (SPSS) 18.0. As associações foram avaliadas com teste do qui-quadrado e com o teste $t$ para comparação de médias aritméticas. Um valor de $\mathrm{p}<0,05$ foi considerado estatisticamente significativo.

\section{Discussão}

A maioria dos pacientes deste estudo foi do sexo masculino (3,7:1), com predomínio na faixa etária de 20-40 anos de idade. A média de idade entre as mulheres com fratura vertebral (30,7 anos) foi menor que a dos homens (34,2 anos), no entanto a diferença não foi estatisticamente significativa, segundo o teste $T$ para comparação entre médias aritméticas (Figuras 1, 2 e 3). Dados condizentes com a literatura vigente e explicados pelo fato de os homens jovens se exporem mais às atividades laborativas e recreativas de risco e serem os principais envolvidos em comportamentos violentos. ${ }^{1,9-11}$

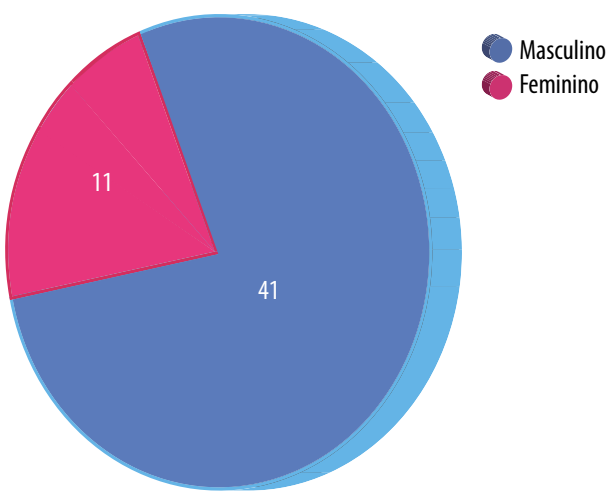

Figura 1 - Distribuição segundo o sexo das fraturas toracolombares tratadas cirurgicamente no Serviço de Neurocirurgia do HBDF entre julho de 2007 e julho de 2012 Fonte: Serviço de Arquivo Médico do Hospital de Base do Distrito Federal (Brasília/Brasil).

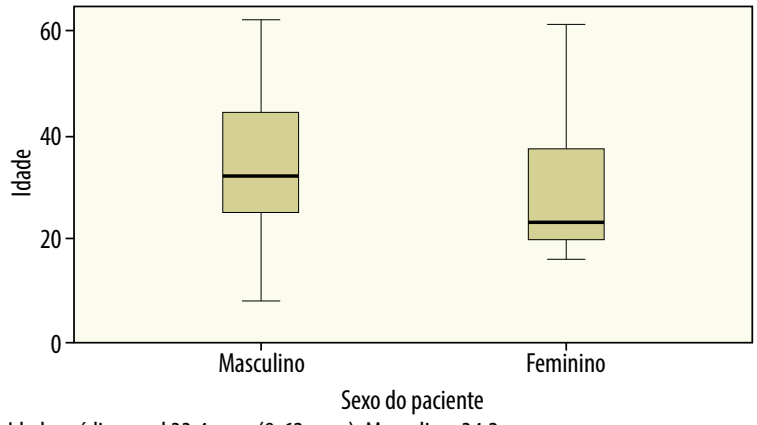

Idade média: geral 33,4 anos (8-62 anos). Masculino: 34,2 anos. Feminino: 30,7 anos ( $p=0,447$ teste $\mathrm{T}$ ).

Figura 2 - Distribuição segundo o sexo e a idade das fraturas toracolombares tratadas cirurgicamente no Serviço de Neurocirurgia do HBDF entre julho de 2007 e julho de 2012. Fonte: Serviço de Arquivo Médico do Hospital de Base do Distrito Federal (Brasília/Brasil).

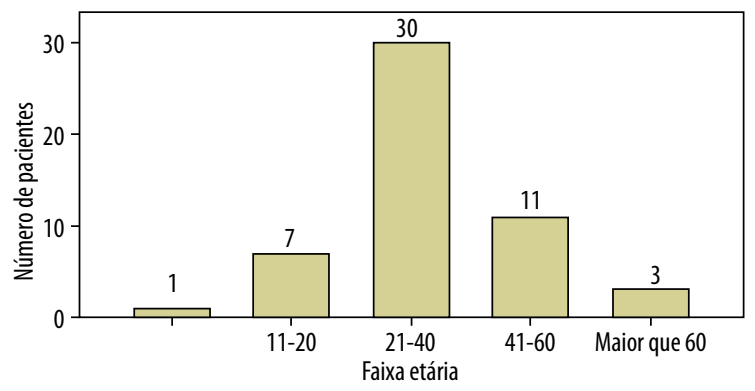

Figura 3 - Distribuição segundo a faixa etária das fraturas toracolombares tratadas cirurgicamente no Serviço de

Neurocirurgia do HBDF entre julho de 2007 e julho de 2012. Fonte: Serviço de Arquivo Médico do Hospital de Base do Distrito Federal (Brasília/Brasil).

Cerca de $48,1 \%$ dos pacientes deste trabalho apresentaram algum déficit neurológico à admissão hospitalar, de acordo com a classificação de ASIA/Frankel (Figura 4), valor muito elevado quando comparado aos dados da literatura. Wyndaele e Wyndaele ${ }^{12}$ apresentaram 35\% dos pacientes com algum grau de comprometimento. Pereira et al. ${ }^{3}$ encontraram incidência de $4,28 \%$. Rodrigues et al. ${ }^{5}$ evidenciaram $11 \%$. De modo geral, a literatura demonstra incidência que varia de $15 \%$ a $40 \%$ de pacientes com fratura da coluna torácica e lombar com lesão neurológica associada. ${ }^{13-15}$

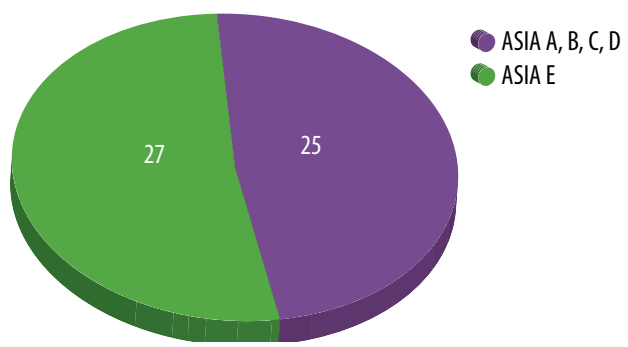

Figura 4 - Distribuição segundo o déficit neurológico das fraturas toracolombares tratadas cirurgicamente no Serviço de Neurocirurgia do HBDF entre julho de 2007 e julho de 2012. Fonte: Serviço de Arquivo Médico do Hospital de Base do Distrito Federal (Brasília/Brasil). 
O segmento vertebral mais acometido na coluna torácica e lombar foi T11-L2 (Figura 5); dados semelhantes aos encontrados por Rodrigues et al. ${ }^{5}$, Reinhold et al. ${ }^{16} \mathrm{e}$ Ning et al. ${ }^{10}$ Segundo Pereira et al. ${ }^{3}$ e Defino et $a l .,{ }^{1}$ é consenso que as vértebras mais acometidas estão na transição toracolombar. A coluna torácica e lombar possui três regiões anatômicas e biomecânicas distintas: coluna torácica alta (T1-T10), junção toracolombar (T11-L2) e coluna lombar (L3-L5). A junção toracolombar é particularmente suscetível a lesões. Fraturas nesse nível representam $50 \%$ de todas as lesões na coluna, excetuando-se as ocorridas na coluna cervical. A transição de um segmento firme (coluna torácica alta) para um móvel (coluna lombar) cria um elevado estresse na junção. A mudança da cifose torácica para lordose lombar deixa a transição suscetível a cargas axiais. ${ }^{3}$

No trabalho de Magerl et al., ${ }^{6}$ a maior parte das fraturas torácicas e lombares era do tipo A. Segundo Vaccaro et al., ${ }^{17}$ a maioria das fraturas por compressão ocorre entre T11 e L2. Com relação à classificação AO, no trabalho original de Aebi et al., ${ }^{18}$ as fraturas do tipo A perfazem um total de $66,16 \%$, as fraturas do tipo B representam $14,4 \%$ dos casos e as do tipo C, 19,38\%. O presente estudo confirma o predomínio das fraturas do tipo A; o segundo grupo mais observado foi o tipo B e em terceiro, o tipo C (Figura 6).

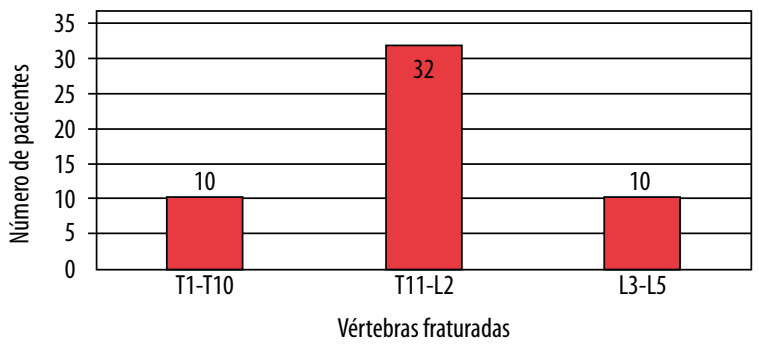

Figura 5 - Distribuição segundo o segmento vertebral das fraturas toracolombares tratadas cirurgicamente no Serviço de Neurocirurgia do HBDF entre julho de 2007 e julho de 2012. Fonte: Serviço de Arquivo Médico do Hospital de Base do Distrito Federal (Brasília/Brasil).

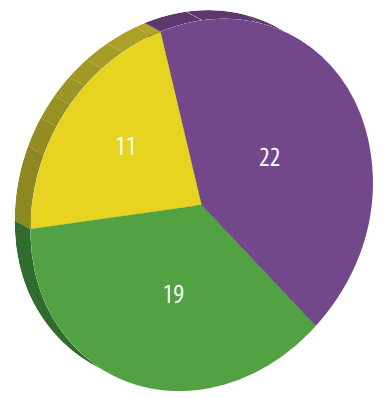

- Tipo A

- Tipo B

Tipo C

Figura 6 - Distribuição segundo a classificação da AO das fraturas toracolombares tratadas cirurgicamente no Serviço de Neurocirurgia do HBDF entre julho de 2007 e julho de 2012. Fonte: Serviço de Arquivo Médico do Hospital de Base do Distrito Federal (Brasília/Brasil).
Os acidentes de trânsito (automobilísticos e motociclísticos) foram as principais causas de fraturas, seguidas por quedas de altura (Figura 7). Achados que são semelhantes aos encontrados no estudo sueco de Jansson et al. ${ }^{19}$ No entanto, a maioria dos outros trabalhos, principalmente as séries brasileiras, mostram a queda de altura como o mais comum mecanismo do trauma. ${ }^{3,9,15,20}$ Esses achados dependem das características demográficas, habitacionais e principalmente socioeconômicas de cada região. ${ }^{5,21,22}$

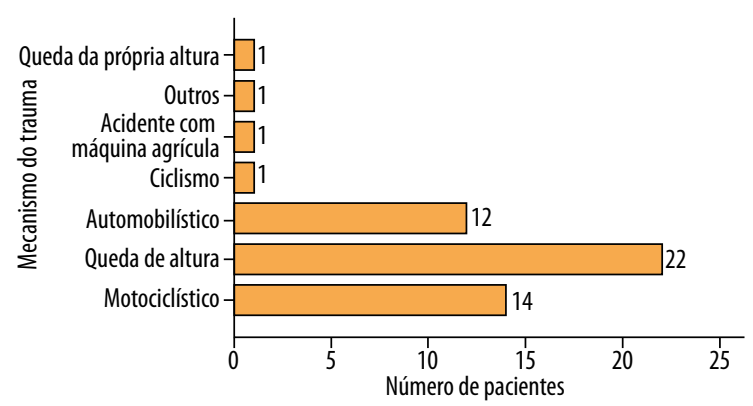

Figura 7 - Distribuição segundo o mecanismo do trauma das fraturas toracolombares tratadas cirurgicamente no Serviço de Neurocirurgia do HBDF entre julho de 2007 e julho de 2012. Fonte: Serviço de arquivo médico do hospital de base do Distrito Federal (Brasília/Brasil).

A maior parte das fraturas torácicas tratadas cirurgicamente foi do tipo rotação $(\mathrm{C}$ da $\mathrm{AO})$ e lombares tipo compressão (A da AO) (Tabela 1), havendo diferenças estatisticamente significativas nesses resultados. Dados semelhantes foram encontrados no trabalho de Reinhold et al., ${ }^{16}$ no qual as fraturas do tipo B e C eram mais frequentes nas lesões torácicas e as fraturas do tipo A, na coluna lombar. A disposição coronal das facetas torácicas dificulta movimentos de distração e translação, permitindo quase tão somente movimentos de rotação. Talvez seja essa uma possível explicação para o predomínio de fraturas rotacionais (tipo $\mathrm{C}$ da AO) a esse nível. ${ }^{1}$

Entre as fraturas torácicas e lombares o sexo, a faixa etária e o mecanismo do trauma não foram fatores de risco para lesão neurológica, uma vez que as diferenças observadas nas tabelas 2, 3 e 4 não foram estatisticamente significativas.

\begin{tabular}{|c|c|c|c|c|}
\hline \multicolumn{5}{|c|}{$\begin{array}{c}\text { Tabela } 1 \text { - Número de pacientes com fratura } \\
\text { toracolombar segundo a classificação da AO e o } \\
\text { segmento vertebral, tratados no Serviço de Neurocirurgia } \\
\text { do HBDF entre julho de } 2007 \text { e julho de } 2012\end{array}$} \\
\hline \multirow{2}{*}{$\begin{array}{l}\text { Segmento } \\
\text { vertebral }\end{array}$} & \multicolumn{3}{|c|}{ Classificação da AO } & \multirow[t]{2}{*}{ Total } \\
\hline & $\mathbf{A}$ & B & $\mathrm{C}$ & \\
\hline Torácico & 3 & 9 & 10 & 22 \\
\hline Lombar & 19 & 10 & 1 & 30 \\
\hline Total & 22 & 19 & 11 & 52 \\
\hline
\end{tabular}


Tabela 2 - Número de pacientes com fratura toracolombar

e déficit neurológico segundo o sexo, tratados no Serviço de

Neurocirurgia do HBDF entre julho de 2007 e julho de 2012

\begin{tabular}{lccc}
\hline \multirow{2}{*}{ Sexo do paciente } & \multicolumn{2}{c}{ Déficit neurológico } & \\
\cline { 2 - 3 } & $\begin{array}{c}\text { Com déficit } \\
\text { (ASIA A, } \\
\text { B, C, D) }\end{array}$ & $\begin{array}{c}\text { Sem déficit } \\
\text { (ASIA E) }\end{array}$ & \\
\hline Masculino & 22 & 19 & 41 \\
Feminino & 3 & 8 & 11 \\
Total & 25 & 27 & 52 \\
\hline
\end{tabular}

$\mathrm{N}=52 ; \mathrm{p}=0,120$.

Fonte: Serviço de Arquivo Médico do Hospital de Base do Distrito Federal (Brasília/DF/Brasil).

Tabela 3 - Número de pacientes com fratura toracolombar e déficit neurológico segundo a faixa etária, tratados no Serviço de Neurocirurgia do HBDF entre julho de 2007 e julho de 2012

\begin{tabular}{lccc}
\hline \multirow{2}{*}{ Faixa etária } & \multicolumn{2}{c}{ Déficit neurológico } & \\
\cline { 2 - 3 } & $\begin{array}{c}\text { Com déficit } \\
\text { (ASIA A, } \\
\text { B, C, D) }\end{array}$ & $\begin{array}{c}\text { Sem déficit } \\
\text { (ASIA E) }\end{array}$ & \\
\hline & 1 & 0 & 1 \\
$11-20$ & 4 & 3 & 7 \\
$21-40$ & 14 & 16 & 30 \\
$41-60$ & 5 & 6 & 11 \\
$>60$ & 1 & 2 & 3 \\
Total & 25 & 27 & 52 \\
\hline
\end{tabular}

$\mathrm{n}=52 ; \mathrm{p}=0,804$

Fonte: Serviço de Arquivo Médico do Hospital de Base do Distrito Federal (Brasília/DF/Brasil).

\begin{tabular}{|c|c|c|c|}
\hline \multicolumn{4}{|c|}{$\begin{array}{l}\text { Tabela } 4 \text { - Número de pacientes com fratura } \\
\text { toracolombar e déficit neurológico segundo o mecanismo } \\
\text { do trauma, tratados no Serviço de Neurocirurgia } \\
\text { do HBDF entre julho de } 2007 \text { e julho de } 2012\end{array}$} \\
\hline \multirow[b]{2}{*}{ Mecanismo do trauma } & \multicolumn{2}{|c|}{ Déficit neurológico } & \multirow[b]{2}{*}{ Total } \\
\hline & $\begin{array}{c}\text { Com déficit } \\
\text { (ASIA A, } \\
\text { B, C, D) }\end{array}$ & $\begin{array}{l}\text { Sem déficit } \\
\text { (ASIA E) }\end{array}$ & \\
\hline Motociclístico & 9 & 5 & 14 \\
\hline Queda de altura & 9 & 13 & 22 \\
\hline Automobilístico & 7 & 5 & 12 \\
\hline Ciclístico & 0 & 1 & 1 \\
\hline Acidente com máquina agrícola & 0 & 1 & 1 \\
\hline Outros & 0 & 1 & 1 \\
\hline Queda da própria altura & 0 & 1 & 1 \\
\hline Total & 25 & 27 & 52 \\
\hline
\end{tabular}

As fraturas torácicas apresentaram mais pacientes com déficit neurológico que as fraturas lombares tratadas cirurgicamente (Tabela 5). Essas diferenças estatisticamente significativas são corroboradas pela literatura vigente, como o trabalho de Gaspar et al. ${ }^{23}$ que mostraram que, entre os pacientes com lesão medular atendidos no Lar Escola São Francisco, São Paulo, o maior nível da lesão foi o torácico, seguido pelo cervical e lombar. O trabalho de Reinhold et al. ${ }^{16}$ observou que $38,6 \%$ das fraturas torácicas apresentavam déficit neurológico, porcentagem estatisticamente maior do que os $23 \%$ das fraturas toracolombares e $25,7 \%$ das lombares, talvez porque a medula espinhal termine ao nível de L1 e a intumescência lombossacra se localize ao nível torácico e ao menor diâmetro do canal raquimedular e do espaço peridural nessa região, tornando a medula espinhal mais vulnerável às lesões neurológicas. ${ }^{1}$

Tabela 5 - Número de pacientes com déficit neurológico segundo o segmento vertebral fraturado, tratados no Serviço de Neurocirurgia do HBDF entre julho de 2007 e julho de 2012

\begin{tabular}{lccc}
\hline \multirow{2}{*}{ S. Vertebral } & \multicolumn{2}{c}{ Déficit neurológico } & \\
\cline { 2 - 3 } & $\begin{array}{c}\text { Com déficit } \\
\text { (ASIA A, } \\
\text { B, C, D) }\end{array}$ & $\begin{array}{c}\text { Sem déficit } \\
\text { (ASIA E) }\end{array}$ & \\
\hline Torácico & 18 & 4 & 22 \\
lombar & 7 & 23 & 30 \\
Total & 25 & 27 & 52 \\
\hline
\end{tabular}

$\mathrm{n}=52 ; \mathrm{p}<0,05$.

Fonte: Serviço de Arquivo Médico do Hospital de Base do Distrito Federal (Brasília/DF/Brasil).

Com relação à classificação da $\mathrm{AO}$, as fraturas do tipo $\mathrm{C}$ apresentaram maior número de pacientes com déficit neurológico e as do tipo $\mathrm{A}$, menor número de lesados medulares, diferenças matematicamente significativas (Tabela 6), dados semelhantes aos publicados por Reinhold et al. ${ }^{16}$ que encontraram $11,1 \%$ de déficit neurológico entre as fraturas do tipo A, 33,1\% do tipo B e $57,4 \%$ do tipo C. No trabalho de Rodrigues et al., ${ }^{5}$ foram encontradas diferenças significativas nas proporções entre os tipos de fraturas. Frankel A é significativamente maior em fratura tipo C; Frankel E é significativamente maior em fraturas tipo A e B do que em C; e os demais tipos de Frankel não apresentaram diferenças significativas entre os diferentes tipos de fraturas. ${ }^{5}$

\begin{tabular}{|c|c|c|c|}
\hline \multicolumn{4}{|c|}{$\begin{array}{c}\text { Tabela } 6 \text { - Número de pacientes com déficit neurológico } \\
\text { segundo o tipo de fratura toracolombar (classificação } \\
\text { AO), tratados no Serviço de Neurocirurgia do } \\
\text { HBDF entre julho de } 2007 \text { e julho de } 2012\end{array}$} \\
\hline \multicolumn{4}{|c|}{ Déficit neurológico } \\
\hline $\begin{array}{l}\text { Classificação } \\
\text { da AO }\end{array}$ & $\begin{array}{c}\text { Com déficit } \\
\text { (ASIA } \\
\text { A,B,C,D) }\end{array}$ & $\begin{array}{l}\text { Sem déficit } \\
\text { (ASIA E) }\end{array}$ & Total \\
\hline A & 7 & 15 & 22 \\
\hline B & 9 & 10 & 19 \\
\hline $\mathrm{C}$ & 9 & 2 & 11 \\
\hline Total & 25 & 27 & 52 \\
\hline
\end{tabular}

$\mathrm{n}=52 ; \mathrm{p}=0,025$.

Fonte: Serviço de Arquivo Médico do Hospital de Base do Distrito Federal (Brasília/DF/Brasil). 


\section{Conclusão}

A maioria dos pacientes com fraturas torácicas e lombares tratados cirurgicamente, no Serviço de Neurocirurgia do Hospital de Base do Distrito Federal no período de julho de 2007 a julho de 2012, era do sexo masculino e da faixa etária compreendida entre 20-40 anos de idade. Cerca de $48 \%$ dos pacientes apresentavam déficit neurológico à admissão hospitalar e tinham a junção toracolombar (T11-L2) como principal local de ocorrência das fraturas.

A fratura tipo compressão (tipo $\mathrm{A}$ da $\mathrm{AO}$ ) foi mais encontrada entre as lesões lombares e a tipo rotação (tipo $\mathrm{C}$ da $\mathrm{AO}$ ), entre as vértebras torácicas. Os principais mecanismos do trauma foram os acidentes de trânsito, seguidos pelas quedas de altura.

As fraturas torácicas e as lesões do tipo C torácica e lombares são fatores de risco para lesão neurológica entre pacientes com traumatismo vertebral tratados cirurgicamente.

A importância deste estudo está principalmente na demonstração da distribuição segundo o mecanismo do trauma das fraturas torácicas e lombares tratadas no HBDF. Isso deve chamar a atenção dos órgãos responsáveis pelo controle do trânsito e de acidentes no país para campanhas direcionadas nesse sentido e auxiliar os gestores da área da saúde.

\section{Agradecimentos}

Gostaria de aproveitar e agradecer a minha mãe, Neusa Braga Rodrigues Tavares, por todo o esforço, carinho e dedicação na criação e estímulo educacional deste jovem piauiense. Ao meu pai, Diaslano de Souza Tavares, que, com certeza, me protege mesmo após seu falecimento. E também à minha esposa, Francisca das Chagas Sheyla de Almeida Gomes, pelo amor dispensado e pela paciência em me aturar e apoiar ao longo destes cinco anos de residência.

\section{Referências}

1. Defino HLA. Lesões traumáticas da coluna vertebral. São Paulo: Bevilacqua Editora; 2005.

2. Holtz A, Levi R. Spinal cord injury. New York: Oxford University Press; 2010.

3. Pereira AFF, Portela LED, Lima GDA, Carneiro WCG, Ferreira MAC, Rangel TAM, et al. Avaliação epidemiológica das fraturas da coluna torácica e lombar dos pacientes atendidos no Serviço de Ortopedia e Traumatologia do
Hospital Getúlio Vargas em Recife/PE. Coluna/Columna. 2009;8(4):395-400.

4. Cunha FM, Menezes CM, Guimarães EP. Lesões traumáticas da coluna torácica e lombar. Rev Bras Ortop. 2000;35(1/2):17-22.

5. Rodrigues LCL, Bortolleto A, Matsumoto MH. Epidemiologia das fraturas toracolombares cirúrgicas na zona leste de São Paulo. Coluna/Columna. 2010;9(2):132-7.

6. Magerl F, Aebi M, Harms J, Nazarian S. A comprehensive classification of thoracic and lumbar injuries. Eur Spine J. 1994;3(4):184-201.

7. Frankel HL, Hancock DO, Hyslop G. The value of postural reduction in the initial management of closed injuries of the spine with paraplegia and tetraplegia. I. Paraplegia. 1969;7(3):179-92.

8. Campbell WW. DeJong, o exame neurológico. $6^{\mathrm{a}}$ ed. Rio de Janeiro: Guanabara Koogan; 2007.

9. Campos MF, Ribeiro AT, Listik S, Pereira CA, Sobrinho JA, Rapoport A. Epidemiologia do traumatismo da coluna vertebral no Serviço de Neurocirurgia do Hospital Heliópolis, São Paulo, Brasil. Rev Col Bras Cir. 2008;35(2):88-93.

10. Ning GZ, Yu TQ, Feng SQ, Zhou XH, Ban DX, Liu Y, et al. Epidemiology of traumatic $\mathrm{SCl}$ in Tianjin, China. Spinal Cord. 2011;49(3):386-90.

11. Vasconcelos ECLM, Riberto M. Caracterização clínica e das situações de fratura da coluna vertebral no município de Ribeirão Preto, propostas para um programa de prevenção do trauma raquimedular. Coluna/Columna. 2011;10(1):40-3.

12. Wyndaele M, Wyndaele JJ. Incidence, prevalence and epidemiology of spinal cord injury: what learns a worldwide literature survey? Spinal Cord. 2006;44(9):523-9.

13. Bucholz RW, Heckman JD. Rockwood e Green: fraturas em adultos. $5^{\mathrm{a}}$ ed. São Paulo: Manole; 2006.

14. Kriek JJ, Govender S. AO-classification of thoracic and lumbar fractures - reproducibility utilizing radiographs and clinical information. Eur Spine J. 2006;15(8):1239-46.

15. Koch A, Graells XS, Zaninelli EM. Epidemiologia de fraturas da coluna de acordo com o mecanismo de trauma: análise de 502 casos. Coluna/Columna. 2007;6(1):18-23.

16. Reinhold M, Knop C, Beisse R, Audigé L, Kandziora F Pizanis A, et al. Operative treatment of 733 patients with acute thoracolumbar spinal injuries: comprehensive results from the second, prospective, Internet-based multicenter study of the Spine Study Group of the German Association of Trauma Surgery. Eur Spine J. 2010;19(10):1657-76.

17. Vaccaro AR, Kim DH, Brodke DS, Harris M, Chapman $\mathrm{J}$, Schildhauer T, et al. Diagnosis and management of thoracolumbar spine fractures. Instr Course Lect. 2004;53:359-73.

18. Aebi M, Thalgott JS, Webb JK, editors. AO ASIF principles in spine surgery. Berlin: Springer; 1998.

19. Jansson KA, Blomqvist P, Svedmark P, Granath F, Buskens $\mathrm{E}$, Larsson $\mathrm{M}$, et al. Thoracolumbar vertebral fractures in Sweden: an analysis of 13,496 patients admitted to hospital. Eur J Epidemiol. 2010;25(6):431-7.

20. Gonçalves AMT, Rosa LN, D'Angelo CT, Savordelli CL, Bonin GL, Squarcino IM, et al. Aspectos epidemiológicos da lesão medular na área de referência do Hospital Estadual Mário Covas. Arq Med ABC. 2007;32(2):64-6.

21. Sekhon LH, Fehlings MG. Epidemiology, demographics, and pathophysiology of acute spinal cord injury. Spine (Phila Pa 1976). 2001;26(Suppl 24):S2-12.

22. Stover SL, Fine PR. The epidemiology and economics of spinal cord injury. Paraplegia. 1987;25(3):225-8. 
23. Gaspar AP, McNeill-Ingham SJ, Vianna PCP, Santos FPE, Chamlian TR, Puertas EB. Avaliação epidemiológica dos pacientes com lesão medular atendidos no Lar Escola São Francisco. Acta Fisiatr. 2003;10(2):73-7.
Endereços para correspondência

Cléciton Braga Tavares

SQS 303, bloco A, ap. 206, Asa Sul

70336-010 - Brasília, DF, Brasil

Conjunto IAPEP, bloco C, ap. 32, Bairro Ilhotas

64015-040 - Teresina, PI

Telefones: (61) 8187-9572; (86) 3223-0191; (86) 9935-7513

E-mail: bragatavares@yahoo.com.br 